\title{
Asymmetric integrality with dimensions of visual pattern
}

\author{
JAMES R. POMERANTZ and LAWRENCE C. SAGER \\ The Johns Hopkins University, Baltimore, Maryland 21218
}

\begin{abstract}
Subjects performed speeded classification tasks with visual patterns that varied in two dimensions: the elements used to construct the patterns (the letters $X, Y, V$, and 0 ) and the configurations formed by the spatial arrangement of the elements. Neither dimension could be attended to selectively without interference from the other, indicating that the dimensions are integral. But the amount of interference between the two dimensions was asymmetrical; irrelevant variation of elements (while classifying by configuration) was harder to ignore than irrelevant variation of configuration (while classifying by elements). This held true whether the element or the configuration discrimination was easier in tasks with no irrelevant variation. It is concluded that the asymmetry is due to attentional strategies in the processing of these patterns and not to the discriminabilities of the dimensions used.
\end{abstract}

In a common form of information-processing task, the subject is presented with a multidimensional stimulus and is required to make a speeded judgment about one of the dimensions of the stimulus. When the set of alternative stimuli varies along dimensions which are irrelevant to the subject's judgment, it is often to his advantage to filter or ignore those irrelevant dimensions and to selectively attend to the relevant one only. Successful selective attention in such tasks is defined by classification performance being independent of variation in the irrelevant dimension.

A now-large body of research has been amassed (Garner, 1974) testing the ease with which certain stimulus dimensions can be filtered. For certain pairs of dimensions, such as the value and chroma of a color chip. subjects are not able to selectively attend to either of the two dimensions effectively: variation in the irrelevant dimension causes interference in discrimination performance. Such pairs of dimensions have been termed "integral" (Garner \& Felfoldy, 1970). With other pairs of dimensions, such as the color and form of a single stimulus, efficient selective attention to either dimension is possible (Gottwald \& Garner, 1972): variation in the irrelevant dimension has no effect on performance. These pairs of dimensions are called "separable."

Typically. dimensions combined to form such compound stimuli have shown symmetrical effects on

This research was supported in part by Grant MH 14229 from the National Institute of Mental Health to Yale University and in part by Biomedical Sciences Support Grant 5S05 RR 07041-09 awanded to The Johns Hopkins University by the Division of Research Resources, DHEW. The authors thank W. R. Garner and $H$. Egeth for their comments on an earlier draft of this paper and $M$. Davis and $T$. Evans for assistance in data collection and analysis. Reprint requests should be sent to James Pomerantz, Department of Psychology, The Johns Hopkins University, Baltimore, Maryland 21218. each other in discrimination tasks. That is, for any pair of dimensions, A and B, either A or B could be selectively attended to (separable) or neither A nor B could (integral). A third possibility exists, however, one in which A could be selectively attended to but not B. Such a possibility, termed "asymmetric integrality" (Garner, 1974), has been found with only one pair of stimulus dimensions (Day \& Wood, 1972; Wood, 1974).

Day and Wood used as stimuli synthetic speech sounds, consonant-vowel monosyllables $/ \mathrm{ba} /$ and $/ \mathrm{da} /$. These stimuli varied not only in a linguistic dimension (place of articulation of the stop consonant. b or d) but also in a nonlinguistic dimension (fundamental frequency). They found that subjects could judge the fundamental frequency of a stimulus while ignoring the stop consonant variation, but not vice versa, even though the two dimensions showed equal discriminability when the irrelevant dimension did not vary.

Three lines of explanation can be differentiated to account for this asymmetry. The first is that the asymmetry arose from the fact that one task was linguistic while the other was not. Linguistic decisions may be handled by a different processor (or a different mode of processing with the same processor) than nonlinguistic decisions. Thus, stop consonant and fundamental frequency may be integral dimensions for the linguistic processor (or mode of processing) but separable for the nonlinguistic one. Alternatively, the linguistic processor may be able to make both linguistic and nonlinguistic decisions while the nonlinguistic one can handle only the nonlinguistic decisions.

A second line of explanation holds that processing asymmetries can arise whenever different modes or hierarchically related levels of processing are required for the two dimensions. That is, asymmetries may result not only when the dimensions involve linguistic 
vs. nonlinguistic decisions, but whenever two fundamentally different levels or modes of processing are called for. A third line of explanation (Garner, 1974) holds that the question of perceptual independence of stimulus dimensions can be answered by examining the logical physical relationship of the dimensions without appeal to indirect inferences about modes of processing. For example, with integral dimensions such as value and chroma, one dimension cannot exist in a stimulus without the other: a stimulus possesses both or neither. With separable dimensions, either may exist quite independently of the other. With asymmetrically integral dimensions, the existence of one of the dimensions demands the existence of the other but not vice versa. With Day and Wood's stimuli, this argument would hold that an auditory stimulus may have a fundamental frequency without also having a stop consonant but that any stimulus having a stop consonant must have a fundamental frequency.

\section{Visual Configurations}

The experiment reported here demonstrates a case of asymmetric integrality with visual patterns. ${ }^{1}$ This finding is of interest, not only in clarifying the question of why asymmetries can occur in perceptual processing in general, but also in giving us some insights into how visual configurations are perceived.

The stimuli used in this experiment are shown in Figure 1. These stimuli vary in two dimensions: the particular elements used (in this case, letters) and the configuration which the elements form. Are there any a priori reasons to expect an asymmetrical relationship in the processing of these dimensions? Each of the three classes of explanation of asymmetry presented above can be considered in turn.

First, with regard to the linguistic vs. nonlinguistic processing distinction, neither of these two stimulus dimensions requires linguistic processing, since the levels on each can be discriminated on the basis of purely visual characteristics (Posner \& Mitchell, 1967). Either dimension could be processed linguistically, however, if, e.g., the levels on the dimensions were named prior to discrimination. This possibility would be more likely with the element dimension (since all the elements are nameable letters) than with the configuration dimension, since at least some of the configurations are less readily named than the elements (see Clement, 1964). Thus, the linguistic vs. nonlinguistic argument would predict either no asymmetry or perhaps a slight asymmetry in the direction of elements being easier to ignore than configuration.

Secondly, an argument in terms of levels of processing could be applied to these stimuli, although it is not clear which of the two dimensions of these stimuli is the more fundamental one, i.e., the one which would be processed before or independently of the other one. A case could be made from the tradition of Gestalt psychology that wholistic or configural processing is primary: discrete stimulus elements are integrated by primitive preattentive organizing processes, and the whole or configuration is the primary unit of analysis on which responses are based. Of course, gestalts can be broken down under scrutiny so that their component elements can be attended to, but this analytic process is only a secondary one. Neisser $(1967$, p. 90$)$, in discussing similar part-whole effects in visual perception states, "Observations like these, which cannot be explained by a single level of feature analysis, pose no particular problem if a predominantly global level of analyzers precedes the extraction or construction of details, and can influence its outcome." Thus, configurations could be attended to directly without processing of individual elements, but attending to elements would necessitate the concurrent or prior processing of configurations. From a structuralist viewpoint, however, the opposite prediction would follow: elements are the primary units of sensation, and the perception of wholes is a secondary process, based on the compounding of discrete sensations.

Lastly, Garner's argument concerning the logical status of the dimensions would share the prediction of the structuralist approach; an element can exist in isolation without configuration, but any configuration must by definition be constructed from elements. An argument of this sort would thus predict that elements could be selectively attended to, but not the configurations they form.

The discriminability of the levels of stimulus dimensions is important in determining how easily they may be attended to or ignored. Increasing the discriminability between levels on a dimension will make that dimension easier to process when it is relevant and harder to ignore when it is irrelevant. Similarly, the more discriminable the relevant dimension, the easier the irrelevant dimension will be to ignore (Morgan \& Alluisi, 1967; Garner \& Felfoldy, 1970). Therefore, an outcome in which the more discriminable dimension was harder to ignore would add nothing new and would tell us little about the way in which these particular dimensions were processed. More interesting would be an asymmetry appearing when discriminabilities were matched, or when the more discriminable dimensions were easier to ignore. For this reason, we varied the levels on our two dimensions in an attempt to equate the two for discriminability.

\section{EXPERIMENT I}

\section{Method}

\section{Stimulus Sets}

This experiment was performed with five different stimulus sets, labeled $A$ through $E$ in Figure 1. Each set consisted of four 

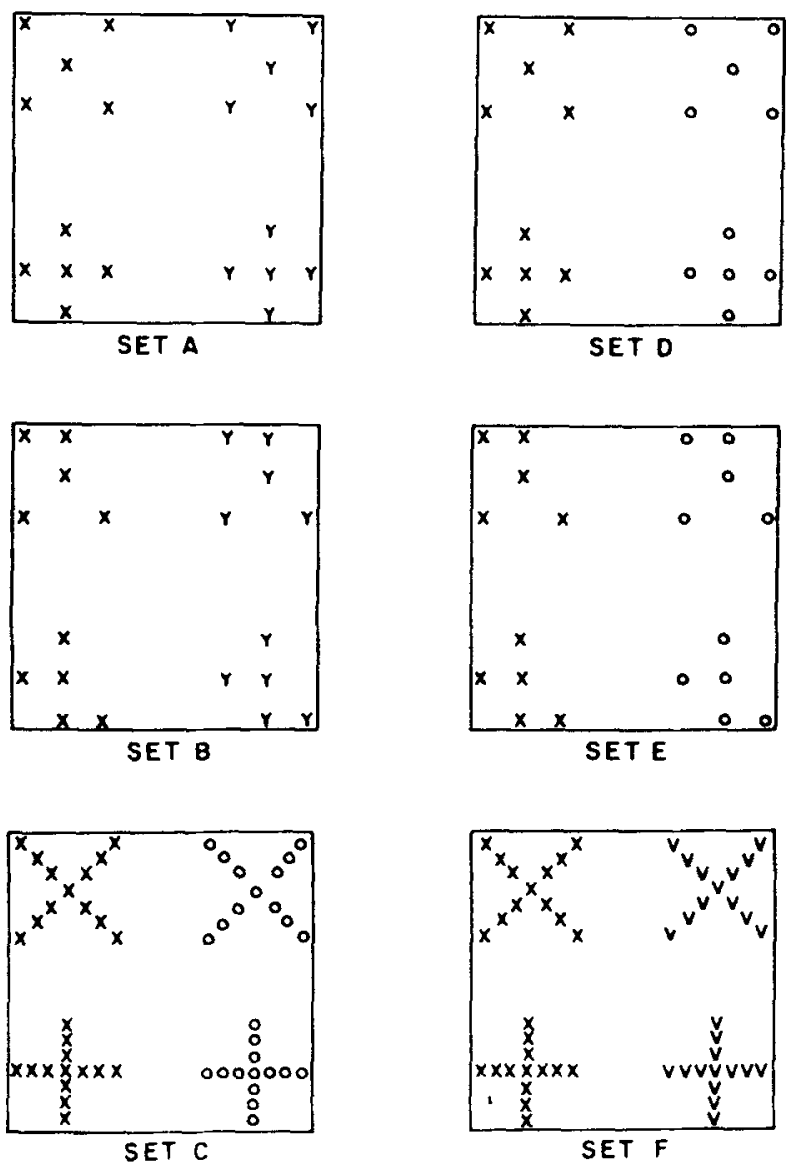

Figure 1. The dx stindhe sets aned in the prentent eqperinents.

different stimuli, formed by crossing two types of elements with two types of configuration. These sets differed with respect to both element and configural discriminability. Element discriminability was varied via the visual similarity of the two letters used: high similarity ( $X$ vs. $Y$ ) in Sets $A$ and $B$ and low similarity ( $X$ vs. $O$ ) in Sets $C, D$, and $E$. Configural discriminability was varied in two ways. The first was pattern goodness (as defined by Garner \& Clement. 1963). Good patterns are known to be more discriminable than poor ones (Clement \& Varnadoe, 1967; Garnet \&Sutliff, 1974). Sets A. C. and D used good patterns. while B and E used poor ones. Secondly. pilot experiments indicated that configurations containing greater numbers of elements were more discriminable than those with fewer elements. Set $C$ used 13-element patterns, while the other sets used 5-element patterns.

\section{Processing Tasks}

Eight different processing tasks were performed with each stimulus set. and these eight were identical in structure for all five sets. The specific tasks for Stimulus Set $\mathbf{A}$ will be described as a representative example. (See Pomerantz \& Garner. 1973, for more detail.)

Single dimension. Four tasks required speeded discrimination between just two stimuli. which varied on only one dimension. Two of these required discriminating the configuration of the stimulus while the element remained constant (at $X$ or $Y$ ) and two required discriminating the element while configuration remained constant at its two respective levels. These four conditions served as control conditions providing baseline performance for each of the two component dimensions while the irrelevant dimension was held constant.

Two correlated dimensions. These tasks. like the four control tasks above. involved discriminating between only two stimuli, but these two stimuli differed in both dimensions; i.e., they differed both in element and in configuration. Since these two dimensions were thus perfectly correlated. both dimensions can be considered relevant, although only one need be processed for successful performance. There were two of these tasks with correlated dimensions, corresponding to the two ways the correlation could be formed.

Two orthogonal dimensioms. These two tasks, unlike the preceding six, involved all four stimuli. In one of the tasks, the subjects were to discriminate among all four on the basis of element, ignoring configuration, while in the other configuration was relevant and elements were to be ignored.

\section{Stimuli and Procedure}

The actual task required in these discriminations was speeded card sorting. Single stimuli were typed onto $6.3 \times 8.9 \mathrm{~cm}$ white cards using uppercase type and black ribbon. At a typical viewing distance of $30 \mathrm{~cm}$, the patterns on the cards subtended a visual angle of about $2.5 \mathrm{deg}$ measured horizontally or vertically. These cards were assembled into decks of 32 cards each, with equal numbers of different stimuli required for each discrimination. Thus, each of the four single-dimension control tasks and the two tasks with correlated dimensions had decks with 16 each of the two component stimuli, while the tasks with orthogonal dimensions used a deck containing eight copies each of the four component stimuli.

In performing each task, the subject was handed a shuffled deck of cards, face up, on top of which were placed exemplars of each of the two (or four) different stimuli in the deck. He placed these exemplars before him to indicate the proper pile into which each alternative stimulus should be placed. All tasks required sorting in to two piles only, one to the left and one to the right. Each subject was allowed to determine the left-right placement of the two piles. The only specific instruction given was the indication of which dimension was relevant on the tasks with orthogonal dimensions (dimensional relevancy was obvious on the control tasks and tasks with correlated dimensions). When the subject was ready, the experimenter gave him the cue to begin sorting by clicking the start button on the stopwatch that timed the subject's performance. The experimenter stopped the watch as the 32 nd card in the deck was placed and then manually recorded the sorting time and any errors committed. General instructions emphasized both speed and accuracy.

Five groups of eight subjects were used, with each group assigned to a different stimulus set. Within each group, each subject performed each of the eight processing tasks once for practice and then eight more times each in mixed blocks. The order in which the tasks were performed was balanced across subjects.

\section{Subjects}

The 40 subjects used, which included 29 males, averaged 23.5 years of age, with a range from 18 to 30 . Most of the subjects were either currently enrolled in or graduated from college. All were paid for serving in a single 1.5 - $\mathrm{h}$ session.

\section{Results}

Mean sorting times for the different processing tasks are given separately for the five stimulus sets in Table 1. Sorting times for the two tasks with correlated dimensions have been pooled within each stimulus set. since the two were formally equivalent and showed virtually identical performance. For the same two reasons. data have been pooled within pairs of single-dimension tasks that differed only in the level used for the irrelevant, constant dimension.

\section{Stimulus Sets A, B, and C}

For Sets A, B, and C, the attempt to match discriminabilities for elements and configurations on the single-dimension tasks was fairly successful. This 
Table 1

Mean Sorting Times for the Different Processing

Tasks in the Three Experiments*

\begin{tabular}{|c|c|c|c|c|}
\hline \multirow[b]{2}{*}{$\begin{array}{c}\text { Dimension } \\
\text { Sorted }\end{array}$} & \multicolumn{3}{|c|}{ Processing Task } & \multirow[b]{2}{*}{$\begin{array}{l}\text { Amount } \\
\text { of Inter- } \\
\text { ference }\end{array}$} \\
\hline & $\begin{array}{c}\text { Single } \\
\text { Dimension }\end{array}$ & $\begin{array}{c}\text { Corre- } \\
\text { lated } \\
\text { Dimensions }\end{array}$ & $\begin{array}{c}\text { Ortho- } \\
\text { gonal } \\
\text { Dimensions }\end{array}$ & \\
\hline & \multicolumn{4}{|c|}{ Experiment I } \\
\hline Stimulus Set $\mathbf{A}$ & & & & \\
\hline $\begin{array}{l}\text { Configuration } \\
\text { Elements }\end{array}$ & $\begin{array}{l}15.29 \\
15.50\end{array}$ & 15.05 & $\begin{array}{l}15.81 \\
15.66\end{array}$ & $\begin{array}{l}.52 \\
.16\end{array}$ \\
\hline Stimulus Set B & & & & \\
\hline $\begin{array}{l}\text { Configuration } \\
\text { Elements }\end{array}$ & $\begin{array}{l}15.48 \\
15.66\end{array}$ & 15.34 & $\begin{array}{l}15.94 \\
15.84\end{array}$ & $\begin{array}{l}.46 \\
.18\end{array}$ \\
\hline Stimulus Set C & & & & \\
\hline $\begin{array}{l}\text { Configuration } \\
\text { Elements }\end{array}$ & $\begin{array}{l}13.28 \\
13.41\end{array}$ & 13.09 & $\begin{array}{l}14.03 \\
13.76\end{array}$ & $\begin{array}{l}.75 \\
.35\end{array}$ \\
\hline Stimulus Set D & & & & \\
\hline $\begin{array}{l}\text { Configuration } \\
\text { Elements }\end{array}$ & $\begin{array}{l}13.90 \\
12.46\end{array}$ & 12.44 & $\begin{array}{l}15.26 \\
12.85\end{array}$ & $\begin{array}{r}1.36 \\
.39\end{array}$ \\
\hline \multicolumn{5}{|l|}{ Stimulus Set E } \\
\hline $\begin{array}{l}\text { Configuration } \\
\text { Elements }\end{array}$ & $\begin{array}{l}14.20 \\
13.32\end{array}$ & 13.34 & $\begin{array}{l}14.96 \\
13.23\end{array}$ & $\begin{array}{r}.76 \\
-.09\end{array}$ \\
\hline Stimulus Set C & \multicolumn{4}{|c|}{ Experiment II } \\
\hline Configuration & 13.00 & & 14.25 & 1.25 \\
\hline \multirow[t]{2}{*}{ Elements } & 13.35 & & 14.21 & .86 \\
\hline & \multicolumn{4}{|c|}{ Experiment III } \\
\hline Stimulus Set F & & & & \\
\hline Configuration & 14.21 & & 15.43 & 1.22 \\
\hline Elements & 14.97 & & 15.87 & .90 \\
\hline
\end{tabular}

*Entries are times (in seconds) required to sort a deck of 32 cards into two piles. Amount of interference was computed by subtracting times with single dimensions from times with orthogonal dimensions.

was not true for Sets $\mathrm{D}$ and $\mathrm{E}$, and they will be discussed briefly below. The general pattern of results for the different processing tasks was identical for Sets $A, B$, and $C$. Considering first the single-dimension tasks, sorting by element with configuration constant was slightly slower (.17 sec per deck) than sorting by configuration with element constant. This difference did not reach significance, however $[\mathrm{F}(1,21)=2.86$, $p>.10]$, and 9 of 24 subjects showed the opposite effect. Thus, the discriminabilities of the two dimensions were fairly closely matched. Standard errors of sorting times for these single-dimension tasks were nearly identical.

These single-dimension tasks serve as controls and provide a baseline against which to measure performance in the conditions with orthogonal. dimensions. The loss in sorting speed in the orthogonal conditions relative to the controls measures the degree to which subjects were unable to ignore the irrelevant dimension. Accordingly, the amount of loss shown by each subject was computed for each stimulus dimension. Of the 24 subjects, 22 showed a loss when sorting by configuration $(.57 \mathrm{sec}$ per deck average) and 17 showed a loss when sorting by element $(.23 \mathrm{sec})$. Both losses were significant by one-tailed binomial test $(\mathrm{p}<.001$ and $\mathrm{p}=.032$, respectively). ${ }^{2}$ Thus neither elements nor configuration could be selectively attended to without interference from the other. However, 21 of 24 subjects showed a greater relative loss when sorting by configuration (ignoring elements) that when sorting by elements (ignoring configuration) $(p<.001$, two-tailed binomial). This outcome is apparent in the interaction with Sets $\mathrm{A}, \mathrm{B}$, and $\mathrm{C}$ in Table 1. Thus, with these three sets, configuration was easier to ignore than elements, although the configuration dimension had at least as much discriminability (and perhaps more) as the elements.

The tasks with correlated dimensions can be compared with the single-dimension conditions to see whether the redundancy provided by the second (relevant) dimension improved performance. Such redundancy gains are a defining characteristic of integral dimensions (Garner \& Felfoldy, 1970). Table 1 shows that net redundancy gains were obtained with these dimensions, and they were shown by 20 of the subjects used with these sets $(p=.002$, two-tailed binomial). Moreover, making what Biederman and Checkosky (1970) have argued is a fairer test of comparing performance on the faster of the two conditions with correlated dimensions against the faster of the two single-dimension tasks separately for each subject, 21 of 24 subjects showed a redundancy gain ( $p<.001$, two-tailed binomial). Therefore, it is concluded that redundancy gains did emerge with the dimensions of Sets A, B, and C.

\section{Stimulus Sets D and E}

These two stimulus sets showed a large inequality of discriminability for the two dimensions. Sorting by elements was significantly faster than by configuration. Significantly more loss was suffered in the orthogonal dimensions tasks relative to the control tasks when sorting was done by configuration than when done by elements, and only the former loss was significantly greater than zero. This asymmetry was much more pronounced than that found with Sets A, $B$, and $C$, and this is consistent with the evidence cited above that such asymmetries can be created, or in this case amplified, when the stimulus dimensions possess greatly differing discriminabilities. No significant redundancy gains were found using Biederman and Checkosky's procedure.

As is typical in card-sorting experiments of this type, errors averaged under $1 \%$ and were too few to analyze. ${ }^{3}$

\section{EXPERIMENT II}

The interference effects found in the first experiment, while statistically reliable, were small in absolute magnitude. Thus the danger arises that they stemmed from some experimental artifact, such as 
subject or experimenter bias in favor of an experimental hypothesis. In Experiment 1 , the experimenter started and stopped the watch that timed subjects' sorting performances, and so it is possible that he could inadvertently have biased the results by stopping the watch earlier on those trials where he expected a short sorting time. To guard against a possible artifactual interpretation, a second experiment was conducted in which timing was placed under control of the subject, not the experimenter.

\section{Method}

Experiment II was a partial replication of the first experiment with a different timing procedure, and so only the differences will be noted. Experiment II used only Stimulus Set C, and used only the single-dimension and orthogonal dimensions tasks (i.e., not the correlated dimensions). Thus there were six tasks in all (four single and two orthogonal). Each of 18 new subjects performed each task once for practice and then six more times in mixed blocks, with the order of the tasks completely counterbalanced across subjects. The experimenter determined the left-right placement of the two sorting piles. Timing was done with an electronic clock operated by separate start and stop switches: When the subject was ready, the experimenter initiated a trial by activating his switch which started the clock and provided an audible cue for the subject to begin sorting. When the subject had sorted the last card in the deck, he pressed a telegraph key, located within close reach, which stopped the clock. This procedure was thus analogous to that most commonly used in discrete RT experiments where the experimenter initiates the timing of a trial and the subject terminates it.

\section{Results}

The results of Experiment II are shown in Table 1. As in the first experiment, sorting by configuration was faster than by elements in the single-dimension conditions $[t(r 7)=3.30, p<.01]$. Losses were shown for both dimensions in the orthogonal dimensions task relative to the controls. Again, this loss was greater when sorting by configuration than when sorting by element $[\mathrm{t}(17)=2.12, \mathrm{p}<.05]$, and this effect was shown by 14 of 18 subjects $(\mathrm{p}=.015)$. Thus, the major effects of Experiment I were replicated.

\section{EXPERIMENT III}

Both of the preceding experiments have shown that elements were harder to ignore than configurations, even when the configurations were more discriminable than the elements. These discriminability differences were slight, however. In the third experiment, we tested whether the asymmetry effect observed in the first two experiments would still obtain when the configuration dimension was made much more discriminable than the elements. For this purpose, we used Stimulus Set $F$ shown in Figure 1, Low element discriminability was achieved by using visually similar letters ( $X$ vs. V), while high configural discriminability was achieved by using good patterns containing 13 elements. This experiment followed the procedure of Experiment II, with timing under the control of the subject. A, new group of 18 subjects was used. The results are again shown in Table 1 . The attempt to make the configural discriminability greater than that for elements was successful, with 16 of 18 subjects showing faster sorting by configuration than by element on the single-dimension tasks. This discriminability difference was more than double that found with Set $C$ in Experiment II and five times that found in Experiment I. Losses in the orthogonal tasks relative to the single-dimension controls were significant for both dimensions. Again, however, more interference resulted when elements had to be ignored than when configuration was the irrelevant dimension, for 14 of the 18 subjects $[p=.015$, one-tailed binomial test; $t(17)=1.99, p<.05$, one-tailed test]. Thus, despite the fact that configural variation should have been harder to ignore by virtue of its relatively high discriminability, element variation was still more interfering.

\section{DISCUSSION}

The pattern of results presented above indicates that these two dimensions of visual pattern, elements and the configuration they form, are integral: interference resulted when the dimensions were combined orthogonally and redundancy gains emerged when they were combined in a correlated fashion. Typically, integral dimensions lead to symmetrical interference effects when discriminabilities on the two dimensions are matched. The present dimensions showed asymmetrical interference effects, and thus they fall closer to Garner's concept of asymmetric integrality. However, unlike the only previously reported case of asymmetric integrality (Day \& Wood, 1972; Wood, 1974), interference effects were found in both directions (i.e., both dimensions interfered with each other). This outcome suggests that asymmetry, like integrality itself, may exist in varying degrees.

\section{Explanations of Asymmetry}

Two questions can be asked concerning why the element-configuration asymmetry was observed. The first concerns what aspects of these two stimulus dimensions were responsible for the asymmetry, and the second concerns what process or mechanism led to the asymmetry.

Starting with the first question, it is most unlikely that the linguistic vs. nonlinguistic nature of the two dimensions was responsible. If either of these dimensions was processed linguistically, it probably would have been the elements, since they were letters, especially in Stimulus Set B when the configurations were not readily nameable. However, elements were selectively attended to more easily than configuration, the opposite of the corresponding outcome found by Day and Wood (1972). The element-configuration asymmetry is partially consistent both with Garner's argument about the logical relationship between the 
two dimensions and with a levels-of-processing argument from a structuralist (vs. gestalt) viewpoint. The consistency is only partial, since the asymmetry did not take the form of a purely unidirectional interference effect. That is, both dimensions interfered to some extent with each other, a result that does not follow directly from either of these positions.

\section{Processing Mechanisms}

Although the asymmetry effect does not follow directly from a levels-of-processing approach, could any model of this type handle all the existing evidence satisfactorily? Let us assume, with Neisser, that two separate analyzers exist, a global analyzer that processes configuration and a local analyzer that processes elements. It is fruitless to ask which of these two analyzers acts more rapidly, since we have shown that this depends on the relative discriminability of the two dimensions being analyzed (cf. Kolers, 1972). Nevertheless, a strict serial model is possible in-which the dimension whose levels are more discriminable is selected for processing first, and a parallel model is possible in which the processing of the more discriminable dimension is completed first. Any serial or parallel model proposing that there will be more interference from the first dimension processed upon the second dimension than vice versa is unsatisfactory, since experiment III has shown more interference from the slower dimension. By the same token, any model dictating the opposite effect-that the slower dimension will interfere more with the faster one-is unsatisfactory, since results from Stimulus Sets $D$ and $E$ showed that the faster dimension produced more interference than the slower one. This model would also fail to explain the results of Day and Wood (1972). Lastly, no model assuming symmetrical interference between the faster and slower dimension can predict any asymmetrical effects. In short, since the element dimension was harder to ignore both when it was processed faster and more slowly than configuration, no model, serial or parallel, based solely on speed or order of processing can handle all of the data.

\section{Attentional Strategies}

The models considered above all assume that the time taken to process a particular stimulus dimension is independent of the task context and of strategies adopted by subjects to perform these tasks. Let us assume, instead, that when a subject attempts to ignore or filter an irrelevant dimension, that dimension gets processed more slowly than when he tries to attend to it in tasks when it is the relevant dimension. Processing speed would still be determined in part by the discriminability of the dimension (i.e., the similarity of the levels employed), but this speed could be further determined by how the subject allocated his attention to the dimension.
Suppose that the subject can vary the size of his attentional field in encoding visual patterns; that is, he can distribute his attention in a wide or narrow beam. Assume that when configuration was the relevant dimension he attended to the entire stimulus, not just a small section of it. (While it would be possible for him to have discriminated the configurations by attending just to the presence or absence of an element in a particular location, Clement and Weiman, 1970, have demonstrated that subjects do not do this, even when so instructed, but rather attend to the entire pattern; see also Sekuler and Abrams, 1968.) Therefore, variation in both configuration and elements would have fallen within the attentional field, opening the way for perceptual interactions and interference. Assume, however, that when elements were the relevant dimension, the subject focused his attention on just one location common to both configurations, since only one element need have been attended to for proper responding. Doing so slowed down the processing of configural information and reduced its interfering effect on processing elements. A model of this sort appears, in principle, to be capable of explaining the major results of the above experiments. Similar models. with different attentional mechanisms could also be applied to separable, fully integral, and unidirectional asymmetrically integral dimensions. Experimental manipulations that act to influence the choice or efficiency of attentional strategies will be required to evaluate such models.

\section{REFERENCE NOTE}

Brownell. H. H. Levels of discriminability in the Stroop test. Unpublished manuscript, The Johns Hopkins University, 1975.

\section{REFERENCES}

Btederman, I., \& Checkosky, S. F. Processing redundant information. Journal of Experimental Psychology, 1970, 83, 486-490.

Clement, D. E. Uncertainty and latency of verbal naming responses as correlates of pattern goodness. Joumal of Verbal Learning and Verbal Behavior, 1964, 3, 150-157.

Clement, D. E.. \& Varnadoe, K. W. Pattern uncertainty and the discrimination of visual patterns. Perception \& Psychophysics, 1967, 2, 427-431.

Clement, D. E., \& Weiman, C. F. R. Instructions, strategies, and pattern uncertainty in a visual discrimination task. Perception \& Psychophysics, 1970. 7, 333-336.

DAY, R. S. \& WOOD, C. C. Interactions between linguistic and nonlinguistic processing. Journal of the Acoustical Society of America, 1972, 51, 79.

GARNER, W. R. The processing of information and structure. Potomac, Md: Lawrence Erlbaum, 1974.

Garner, W. R., \& Clement, D. E. Goodness of pattern and pattern uncertainty. Journal of Verbal Learning and Verbal Behavior, 1963, 2, 446-452.

GARNER, W. R., \& FElfoldy, G. L. Integrality of stimulus dimensions in various types of information processing. Cognitive Psychology, 1970, 1, 225-241. 
Garner. W. R.. \& Sutliff. D. The effect of goodness on encoding time in visual pattern discrimination. Penception \& Psychophysics. 1974, 16. 426-430.

GotTwald. R. L.. \& GARNER, W. R. Effects of focusing strategy on speeded classification with grouping. filtering and condensation tasks. Perception \& Psychophysics. 1972. 11. 179-182.

Jensen. A. R.. \& Rohwer. W. D. The Stroop color-word test: A review. Acta Psychologica. 1966, 25. 36-93.

Kolers. P. A. Some problems of classification. In J. F. Kavanagh \& I. G. Mattingly (Eds.), Language by ear and by eye. Cambridge. Mass: M.I.T. Press. 1972.

Morgan. B. B.. \& Alcursi. E. A. Effects of discriminability and irrelevant information on absolute judgments. Perception \& Psy.chophysics. 1967. 2. 54-58.

NeIsser. U. Cognitive psychology. New York: Appleton-CenturyCrofts. 1967.

Pomerantz. J. R.. \& Garner. W. R. Stimulus configuration in selective attention tasks. Perception \& Psychophysics. 1973. 14. 565-569.

Posner. M. I., \& Mrtchell, R. F. Chronometric analysis of classification. Psychological Review, 1967, 74, 392-409.

Sexuler, R. W., \& Abrams, M. Visual sameness: A choice time analysis of pattern recognition processes. Joumal of Experimental Psychology, 1968, 77, 232-238.

Wood. C. C. Parallel processing of auditory and phonetic information in speech discrimination. Perception \& Psychophysics. 1974. 15. 501-508.

\section{NOTES}

1. It could be argued that the Stroop test. which measures interference between visually presented words and the ink color in which they are printed, provides another instance of asymmetry, since there is less interference of ink color on responses to words than vice versa (Jensen \& Rohwer, 1966). However, H. Brownell (Note 1 ) has show $n$ that the direction of this asymmetry depends on which dimension. ink color or word. is made more discriminable. If it were possible to match discriminabilities precisely, the asymmetry would presumably disappear. Also. these two dimensions fail to show characteristic redundancy gains when combined in a correlated fashion and so are not integral by Garner's definition.

2. One-tailed tests are appropriate when comparing performance in conditions with orthogonal dimensions with that on control conditions. since the added irrelevant information provided with orthogonal dimensions could only impair performance, not systematically improve it.

3. Unlike in most discrete RT tasks. it is possible in card sorting for the subject to correct a response by moving a misplaced card over to the correct pile. In fact. subjects seldom make errors that they do not detect and correct immediately, so few errors remain at the end of each sorting to be counted. Thus, errors that are corrected actually add to sorting times. This makes it very unlikely that subjects would vary speed/accuracy tradeoffs between tasks. since attempting to speed up would result in more errors to be corrected, which would ultimately slow performance down.

(Received for publication June 30. 1975; revision received August 27. 1975.) 